\title{
Estimation of Fiber Diameters in the Spinal Dorsal Columns from Clinical Data
}

\author{
Wilbert A. Wesselink,* Jan Holsheimer, Bart Nuttin, Herman B. K. Boom, Senior Member, IEEE, \\ Gary W. King, Jan M. Gybels, and Paul de Sutter
}

\begin{abstract}
Lack of human morphometric data regarding the largest nerve fibers in the dorsal columns (DC's) of the spinal cord has lead to the estimation of the diameters of these fibers from clinical data retrieved from patients with a new spinal cord stimulation (SCS) system. These patients indicated the perception threshold of stimulation induced paresthesia in various body segments, while the stimulation amplitude was increased.

The fiber diameters were calculated with a computer model, developed to calculate the effects of SCS on spinal nerve fibers. This computer model consists of two parts: 1) a three-dimensional (3-D) volume conductor model of a spinal cord segment in which the potential distribution due to electrical stimulation is calculated and 2) an electrical equivalent cable model of myelinated nerve fiber, which uses the calculated potential field to determine the threshold stimulus needed for activation.

It is shown that the largest fibers in the medial DC's are significantly smaller than the largest fibers in the lateral parts. This finding is in accordance with the fiber distribution in cat, derived from the corresponding propagation velocities. Moreover, it is shown that the mediolateral increase in fiber diameter is mainly confined to the lateral parts of the DC's. Implementation of this mediolateral fiber diameter distribution of the DC's in the computer model enables the prediction of the recruitment order of dermatomal paresthesias following increasing electrical stimulation amplitude.
\end{abstract}

Index Terms-Computer modeling, dorsal columns, fiber diameter, spinal cord stimulation.

\section{INTRODUCTION}

$\mathbf{S}$ PINAL cord stimulation (SCS) for the management of chronic pain is based on the "gate control" theory [1]. It is assumed that the inhibition of small diameter noxious pathways is modulated synaptically by large diameter somatosensory $(\mathrm{A} \beta)$ nerve fibers. Suppression of chronic pain can be achieved by stimulation of these large fibers in the dorsal columns (DC's) or dorsal roots (DR's) of the spinal cord. It is accompanied by paresthesia, which may cover those dermatomes corresponding to the spinal cord level of the cathode down to the caudal end.

Manuscript received July 25, 1997; revised May 11, 1998. This work was supported by a Grant from Medtronic Inc., Minneapolis, MN. Asterisk indicates corresponding author.

*W. A. Wesselink is with the Institute for Biomedical Technology, University of Twente, P. O. Box 217, 7500 AE Enschede, The Netherlands (e-mail: w.a.wesselink@el.utwente.nl).

J. Holsheimer and H. B. K. Boom are with the Institute for Biomedical Technology, University of Twente, 7500 AE Enschede, The Netherlands.

B. Nuttin, J. M. Gybels, and P. de Sutter are with the Department of Neurosurgery, University Hospital Gasthuisberg, B-3000 Leuven, Belgium.

G. W. King is with the Medtronic Neurological Division, Minneapolis, MN 55440-9087 USA.

Publisher Item Identifier S 0018-9294(98)07782-9.
To achieve a better understanding of the physical basics of SCS, a computer model has been developed at the University of Twente, the Netherlands [2], [3]. This UT-SCS model enables the simulation of spinal nerve fiber responses to electrical stimulation by epidurally placed electrodes. For some years, the model has been used for the development of new electrodes, which may give improved paresthesia coverage of painful areas and a decrease of unwanted side effects [4]-[6]. Although most essential parameters required for the model are known fairly well from literature, the diameter distribution of the A $\beta$ fibers in the superficial layers of the DC's of the human spinal cord, where these fibers are most probably activated, is still unknown. Prediction of the recruitment order of the paresthesia areas, while increasing the stimulus amplitude, will only be possible when the positions as well as the diameters of the corresponding fibers in the DC's have been determined.

The topography of the fibers in the DC's is only roughly known. Anatomical studies on human and animal spinal cord have shown that generally the topographical organization of the DC's has the form of a fiber lamination, i.e., fibers corresponding to different body segments are lying in parallel layers, approximately normal to the dorsal border of the DC's. The dorsomedial positions are occupied by DC fibers arising from the most caudal DR fibers, whereas fibers originating from more rostral levels join laterally [7]-[15]. No direct functional association has been shown to this structure. At cervical levels, a transformation has been found from a segmental fiber arrangement into a more functionally oriented fiber arrangement, which also has a closer similarity to the representation in the cortex [10]-[12], [14]. To determine the diameters of the fibers, it was assumed that the largest fibers at the surface of the DC's determine the perception threshold of paresthesia of the corresponding dermatomes.

Studies on cats showed that the conduction velocity of action potentials in the DC's decreased by $25 \%-70 \%$ from a low-thoracic to a high-cervical level [16]-[20]. For $\mathrm{A} \beta$ fibers specifically, a decrease in conduction velocity of $35 \%$ was reported [16]. Since DC fibers enter the lateral part of the DC's and then gradually shift medially while ascending, and because conduction velocity is linearly related to fiber diameter, the diameters of DC fibers should decrease from the lateral to the medial parts of the DC's, assuming that these fibers have similar diameters at their entrance into the spinal cord. It has also been reported that a larger decrease in diameter corresponds to a more dense collateral branching of the fiber [18]. Since collaterals arise more frequently in the proximity of the DR entry zone than at a larger distance [21], 
most reduction in diameter of the ascending fiber is expected to occur in the lateral parts of the DC's.

Morphometric studies of myelinated fibers in the DC's of the human spinal cord were performed by Häggqvist [22], Ohnishi et al. [23] and Makino et al. [24]. Häggqvist measured diameters in the fasciculus gracilis and the fasciculus cuneatus at level $\mathrm{T} 3$ ranging from 1 to $15 \mu \mathrm{m}$, whereby $60 \%$ of the fibers were between 1-3 $\mu \mathrm{m}$. It was observed that the largest fibers in the medial DC's were smaller (13-14 $\mu \mathrm{m})$ than the largest fibers more laterally $(14-15 \mu \mathrm{m})$. Ohnishi et al. [23] reported smaller diameters $(1-7 \mu \mathrm{m})$ in the fasciculus gracilis at level T5, with a peak at 2-3 $\mu \mathrm{m}$. Recently, Makino et al. [24] analyzed myelinated fibers in the white matter of the human spinal cord at level C7. In the DC's, diameters were between $2-15 \mu \mathrm{m}$, with a peak at 3-5 $\mu \mathrm{m}$. However, since the results of these studies are based on relatively large areas in the DC's, it is not known whether they give a correct representation of fiber diameters in their superficial layers, whereas any functional association is unclear.

The purpose of the current study was to estimate the diameter distribution of the lowest threshold fibers in the superficial DC's by using clinical data as the input for the UT-SCS model and to associate them with paresthesia areas. These data were obtained from patients enrolled in a study to test a newly designed multiple contact electrode, the transverse tripole (TTL) [25]. Geometrical data of the patient's spinal cord were retrieved from computed tomography (CT) scans, impedance measurements were taken to fit the model impedance, and during (percutaneous) stimulation the patient reported the perception threshold of paresthesia of a number of body segments. Then, while assuming certain positions of the nerve fibers corresponding to these body segments, the diameters of these fibers were calculated from the measured perception thresholds.

By this inverse method, the computer model can be tuned by implementing the calculated distribution of fiber diameters at a specified spine level, and a correct prediction of the recruitment order of paresthesia areas due to stimulation by any specific electrode configuration may be obtained.

\section{METHODS}

\section{A. Clinical Study}

The clinical part of the study was carried out according to a clinical investigation plan designed to evaluate the efficacy of the TTL electrode and to test its ability to steer the electric field with a new external dual channel pulse generator [25]. Selection of patients, implantation of the TTL electrode, and patient testing were performed at the University Hospital "Gasthuisberg" (Leuven, Belgium). The electrodes for this pilot study were custom made by Medtronic (Minneapolis, $\mathrm{MN}$ ). Two versions were used, one with a flat paddle (type A), and one with a curved paddle (type B), designed to fit inside a relatively small vertebral canal. The type used for each patient model is indicated. A schematic drawing of the TTL system is shown in Fig. 1.

The TTL electrode has three contacts in the transverse plane, a central cathode and two lateral anodes [4]. A fourth contact is caudal to the central cathode, and enables longitudinal bipolar

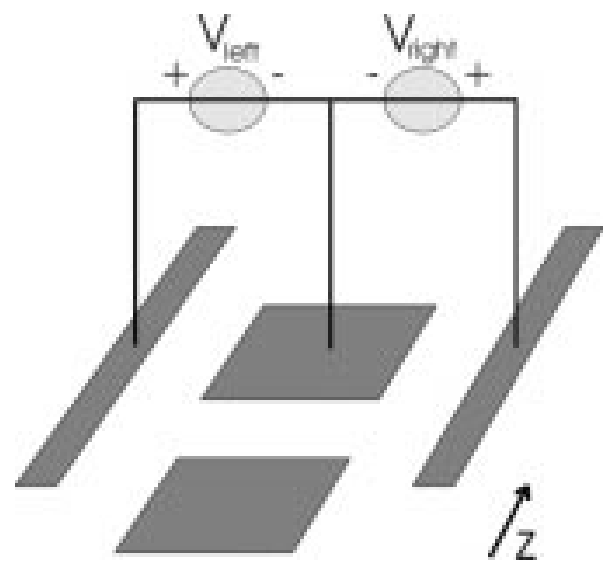

Fig. 1. Schematic drawing of the TTL and the dual channel pulse generator; z: longitudinal direction; see text for dimensions of contacts and their separation.

stimulation. The central contacts are 2-mm long and 3-mm wide, the lateral contacts $6-\mathrm{mm}$ long and $0.6-\mathrm{mm}$ wide, and they are separated by $2.5-3 \mathrm{~mm}$. The cathode stimulates the medial DC fibers, while the anodes increase the stimulation threshold of the laterally situated DR and DC fibers. This electrode can be used in combination with a dual channel, constant voltage pulse generator, generating two simultaneous (positive) pulses at the left and the right lateral anodal contacts with independent amplitude control. The central contact is the common cathode. This dual channel pulse generator has especially been designed for percutaneous testing with this type of electrode. It is, for example, possible to correct for offmidline placement of the electrode by the facility to steer the potential field [4]. This steering can be achieved by changing the amplitude ratio of the left and right pulses. For example, 0.4 left means that the amplitude of the left channel is $40 \%$ of the amplitude of the right channel. A completely unbalanced situation ( 0.0 left) changes the left anode into a cathode. When the electrode was used as a longitudinal bipole, only one channel of the stimulator was connected.

The shape and size of the electrode required a flavectomy for placement in the dorsal epidural space. Prior to the implantation, transverse CT scans of the spinal cord with intradural contrast were made, covering the area where the TTL was to be placed. After electrode implantation a second series of CT scans was made, showing the position of the anodal and cathodal contacts. In this way, the geometrical data of each patient's spinal cord, surrounding tissues and electrode position were retrieved and used to assemble a three-dimensional (3-D) volume conductor model. Prior to complete internalization, two trial screening tests were performed with the (external) dual-channel pulse generator via percutaneous wires. A screening session consisted of measuring the load resistance, longitudinal bipolar stimulation and transverse tripolar stimulation with nine balance settings (1.0 and $0.9,0.7,0.4,0.0$ at both left and right sides). The applied pulse rate was $50 \mathrm{pps}$, the duration of the rectangular pulses was $210 \mu \mathrm{s}$. The patients described the location of paresthesia in standard separate body areas as a function of voltage level. After complete internalization, and at follow up visits of $4,12,26$, and 52 weeks patients were retested, using an 
internal (Itrell II ${ }^{\circledR}$, Medtronic, Minneapolis, MN) or external (X-trel ${ }^{\circledR}$, Medtronic, Minneapolis, MN) single-channel pulse generator. No CT scans were made at these follow-up tests. These pulse generators only allow three balance settings of the transverse tripolar configuration: $1.0,0.0$ left, and 0.0 right. Of all screening sessions, the data of the session just prior to or after the CT scans had been made, were selected for diameter estimation, in order to avoid a change in electrode position due to migration between scanning and testing. Still, the patient was moved from one room to another between scanning and testing and was most probably lying in slightly different positions, possibly introducing small variations of the position of the electrode with respect to the spinal cord (see Section IV).

\section{B. Modeling Study}

The results of the clinical study provided the data necessary to perform the modeling described in this paper estimating the fiber diameters in the superficial DC's. The pre- and post-operative $\mathrm{CT}$ scans were combined to reconstruct a 3-D discretized model of each patient's spinal cord with the UTSCS computer model. The resulting inhomogeneous volume conductor represents the spinal cord gray and white matter, cerebrospinal fluid (CSF), dura mater, epidural tissue, vertebral bone, and a surrounding layer representing more distant tissues. Also included are the geometry of the electrode contacts and surrounding insulating flexible lead material. Fig. 2 shows a transverse section of a patient's volume conductor model of the spinal cord. The model consists of $56 \times 56 \times 56$ elements with variable dimensions $(0.15-4.0 \mathrm{~mm})$. The smallest elements are localized in the area where the largest potential gradients are expected: between the electrode and the outer border of the DC's [3]. According to the voltage ratio at the left and right channel of the stimulator, the potentials of the anodes as referred to the cathode are selected. The three potentials are chosen in such a way that the current leaking to the border of the model (set at $0 \mathrm{~V}$ ) is less than $2 \%$. A finite difference technique is used to obtain the potential field due to the imposed potential differences. The resulting equations are solved using a red-black Gauss-Seidel iteration with variable overrelaxation [26].

The conductivities of the various anatomical structures incorporated in the volume conductor model are presented in Table I. As in a previous validation study [27], the conductivity of the dura mater, including the conductivity of epidural fat which may be present between the electrode contacts and the dura mater, was estimated from load resistance data. Assuming that the resistance is almost completely localized close to the contact-tissue interface [5] (epidural fat and dura mater), the dura mater conductivity in the current study was chosen to match the measured resistances of bipolar and tripolar contact configurations from the corresponding patients. The resistance of each contact was calculated separately from the measurements. Accordingly, the equivalent conductivity of the dura could have a different value near each contact. The calculated potential field in the volume conductor model was used to determine the external nodal membrane potentials of the branched myelinated nerve fibers at specified positions in the DC's. Hereafter, the diameter of the branched DC

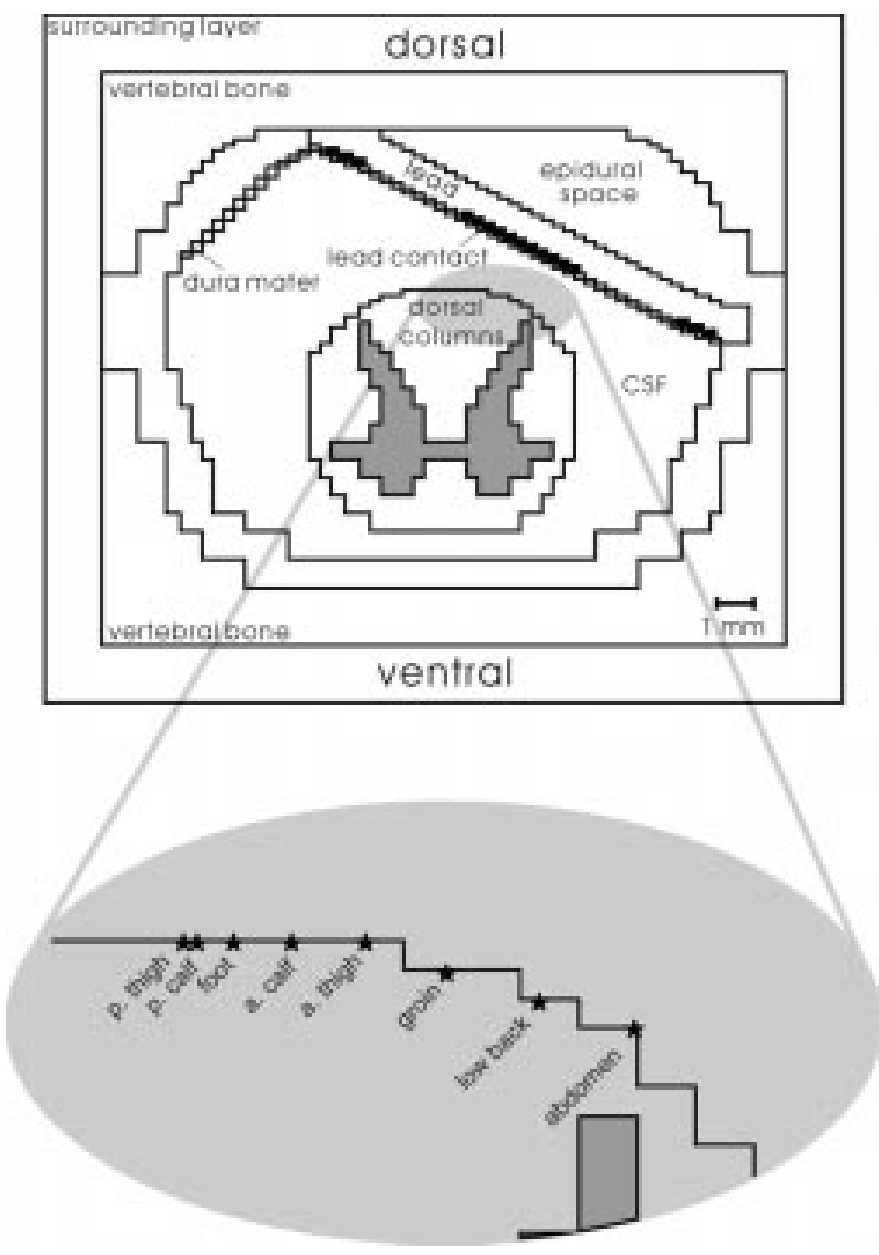

Fig. 2. Transverse section of the 3-D volume conductor model A1; inset: positions of the nerve fibers in one dorsal column; see text for separations between fiber positions.

fiber was calculated such that the perception threshold of the corresponding dermatome matched the activation threshold of the considered fiber, using a pulsewidth of $210 \mu$ s. For this, a McNeal type cable model of myelinated nerve fiber was used [28], extended with collaterals and with all nodes made excitable. Parameters describing the electrical behavior of the nodal membrane were taken from experimental data from Chiu et al. [29], and adjusted for body temperature $\left(37^{\circ} \mathrm{C}\right)$. It has been shown, that the threshold stimulus of a DC fiber is reduced up to $50 \%$, when collaterals into the gray matter with a diameter of $1 / 3$ of the DC fiber were present, but that the number of collaterals hardly influences the threshold stimulus, provided that one is present at the level of the cathode [2]. Therefore, all DC fibers were modeled with the same number of collaterals (8). Moreover, we determined that the conduction velocity of a propagating action potential is hardly influenced by the presence of any (realistic) number of collaterals. An extensive description of the equivalent model of the branched DC nerve fiber has been given by Struijk et al. [2], while the properties of the model of the curved DR fiber are discussed in [3]. See also [30].

Since patients with chronic pain in the lower extremities were selected, the electrodes were implanted at low-thoracic vertebral levels, i.e., T7-T10. The paresthesia coverage was stored using a body map including 69 areas. Due to the level 
TABLE I

Conductivities of the Volume Conductor Model [S/m]

\begin{tabular}{ll}
\hline gray matter & 0.23 \\
white matter & 0.60 longitudinal \\
& 0.083 transverse \\
cerebrospinal fluid & 1.7 \\
epidural fat & 0.04 \\
vertebral bone & 0.02 \\
surrounding layer & 0.004 \\
electrode insulation & 0.002 \\
\hline
\end{tabular}

of stimulation, only 16 body areas could be covered with paresthesia. The precise areas of the corresponding DC fibers are unknown, but the order in which they are represented in the DC's is, from medial to lateral: posterior thigh, posterior calf, foot, anterior calf, anterior thigh, groin, low back, and abdomen [9], [12]-[14]. Due to the reduction of the number of fibers originating from a dermatome when ascending in the spinal cord [7], the width of the laminated dermatomal projections in the DC's decreases from lateral to medial [8], [9], [12]-[14]. Based on these topographical aspects, the order of the fibers in the DC's, each representing the most sensitive fiber of the population corresponding with one of the body segments, was chosen accordingly, and the distance between the fibers was progressively increased from medial to lateral $(0.05,0.2,0.3,0.4,0.4,0.5,0.5 \mathrm{~mm}$, respectively). The inset of Fig. 2 shows the typical positions of the selected fibers in one half of the superficial DC's of a low-thoracic model. In order to analyze the effect of the selected fiber positions on the calculated diameters, the results were compared with those resulting from equidistant fiber positions. Since stimulation thresholds will be lowest for fibers at the surface of the DC's, these fiber positions are assumed to be related to the perception threshold of paresthesia. However, the effect of positioning a fiber deeper in the DC's was also determined.

\section{RESULTS}

Four patients were enrolled in this pilot study in which the transverse tripolar system was applied. Data from three patients were used in the current study, because only their CT scans showed enough detail to enable the reconstruction of a 3-D volume conductor model of the spinal cord and surrounding anatomical structures. The paresthesia coverage enabled the estimation of the fiber diameters related to all body segments below the spine level of the electrode. Because not all body segments necessarily showed paresthesia when different contact configurations were considered, the minimum and maximum number of diameters calculated per fiber are shown in Fig. 3. Migration or wire breakage necessitated repositioning or replacement of electrodes, finally resulting in five separate patient models. The patients will be referred to as $\mathrm{A}, \mathrm{B}$, and $\mathrm{C}$, and their models are numbered 1 or 2 . The conductivities of the dura mater as determined for each patient model are given in Table II. The medial and lateral parts of the dura mater have different values, which might be due to the different amounts of epidural fat between dura mater, and lateral and medial electrode contacts, respectively. In case the calculated diameters of DC fibers, corresponding to the most rostral dermatomes giving paresthesia (most lateral in the DC's), turned out to be rather large, it was assumed that DR fibers were activated instead of DC fibers, and the corresponding DR fiber diameters were calculated. A previous study has already shown that DR fiber activation thresholds are generally lower than those of DC fibers, which is mainly due to the fact that DR fibers are curved and cross the white matter-CSF interface [3].

In patient model A1, the electrode (type A) had an asymmetrical position at level T8, as shown in Fig. 2. The test data were obtained after internalization of the SCS system, using a single channel stimulator (Itrell $\mathrm{II}^{\circledR}$, Medtronic), and comprised five contact configurations: a monopole, a bipole and three tripoles (three balance settings). Fig. 3(a) shows the mean fiber diameters calculated from the five configurations. Horizontally, the fibers are shown in the order they are represented in the DC's (cf. Fig. 2), and indicated by the corresponding body areas. Vertically, the mean fiber diameters and their standard deviations are shown. The values range from 10 to $19 \mu \mathrm{m}$, whereby the mean diameters increase from the medial to the lateral DC's. Only the most left DC fibers (abdomen, low back) deviate from this trend. The rectangle represents the diameter of a DR fiber, when left abdominal paresthesia would be evoked by DR stimulation, and its size nearly equals the size of the DC fiber.

Patient model A2 ensued after repositioning of this electrode. Now the electrode had an almost symmetrical position to the midsagittal plane at level $\mathrm{T} 8$. The test data comprised the same contact combinations as in model A1. The calculated mean fiber diameters are shown in Fig. 3(b). Although an increase in fiber diameter is still present in mediolateral direction, the diameters are much smaller $(1.8-8.1 \mu \mathrm{m})$ than in model A1. Furthermore, the diameters of the two most medial fibers at the left side of the DC's are larger. The calculated DC fiber diameters of the left and right abdomen are extremely large as compared with the other DC fibers. If abdominal paresthesia is supposed to be evoked by stimulation of DR fibers, fiber diameters are significantly smaller, as indicated by the rectangles in Fig. 3(b).

In the third patient model, referred to as B1, the electrode (type A) is nearly symmetrical at level T10. The data are obtained during trial screening, using the external dual channel stimulator (longitudinal bipolar and nine transverse tripolar tests, corresponding to nine balance settings). The calculated mean fiber diameters, presented in Fig. 3(c), range from 10 to $19 \mu \mathrm{m}$. The extremely large DC fiber corresponding to the left low back $(19 \mu \mathrm{m})$ is smaller when it is considered to be a DR fiber $(13 \mu \mathrm{m})$. The most medially positioned fibers are almost equal in size to the most lateral fibers, whereas the fibers related to the feet are approximately $15 \%$ smaller.

After implantation of a new lead (type B) at the same level (T10) the data of patient model B2 were obtained. The position of this lead was symmetrical to the spinal cord and the central cathode was slightly further away from the spinal cord than in model B1. Testing of this electrode was also performed with a dual channel stimulator (a bipolar and a tripolar contact configuration with nine balance settings). The range of calculated diameters is similar to the results of B1, just like the distribution of fiber diameters, as shown in Fig. 3(d). 

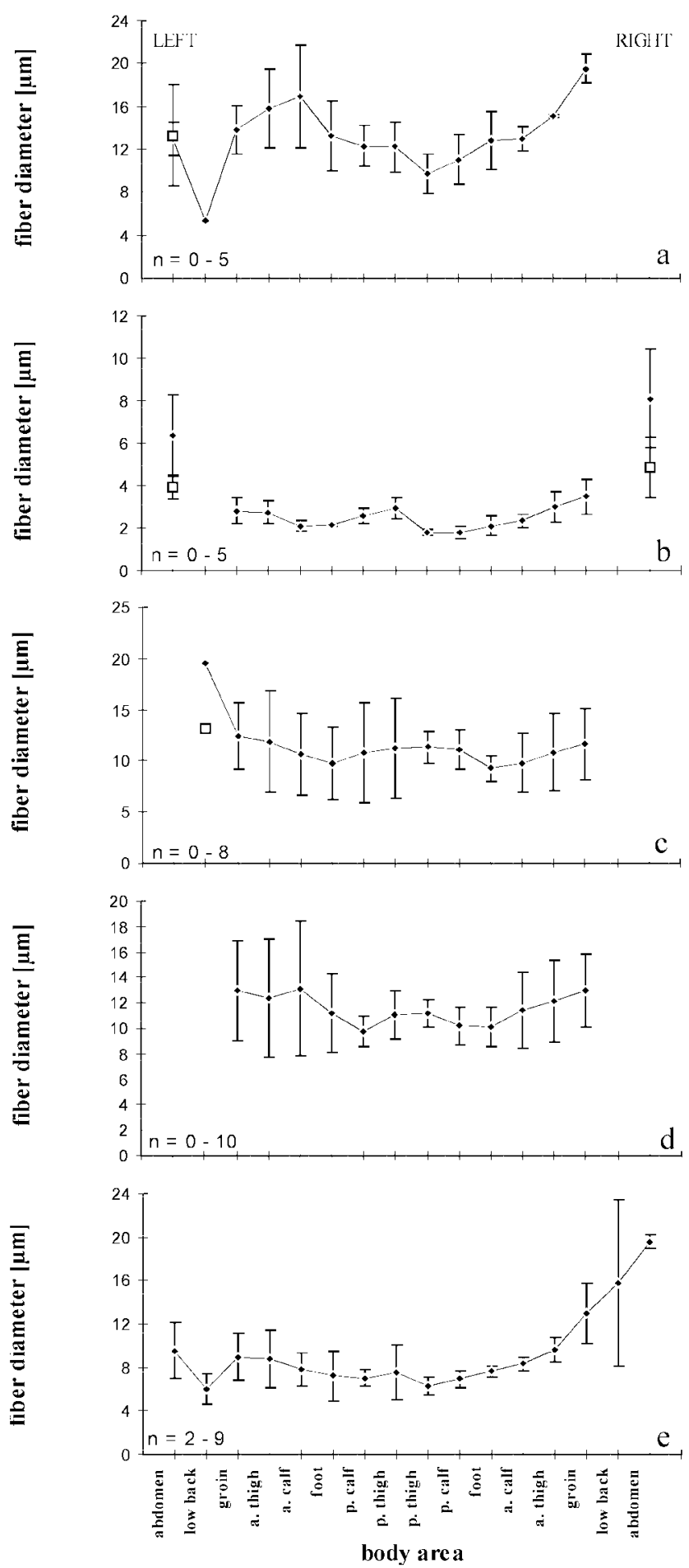

Fig. 3. Fiber size distributions of patient models (a) A1, (b) A2, (c) B1, (d) $\mathrm{B} 2$, and (e) $\mathrm{C} 1$; horizontally: body areas left and right; vertically: calculated mean fiber diameters and standard deviations; $n=$ number of calculated diameters per fiber (min. and max. shown per patient model).

Patient model $\mathrm{C} 1$ is the fifth model used in this study. The electrode (type B) was implanted at T7, slightly left of the midsagittal plane. The test data were obtained from stimulation with the external, dual channel stimulator (tripolar stimulation with nine balance settings). Fig. 3(e) shows the diameter distribution as calculated for this patient model. The mean fiber diameters varied between 7 and $19 \mu \mathrm{m}$. An
TABLE II

Dura Mater Conductivity for Five Patient Models

\begin{tabular}{ccc}
\hline patient model & medial $[\mathrm{S} / \mathrm{m}]$ & lateral $[\mathrm{S} / \mathrm{m}]$ \\
\hline A1 & 0.049 & 0.102 \\
A2 & 0.058 & 0.130 \\
B1 & 0.044 & 0.041 \\
B2 & 0.054 & 0.045 \\
C1 & 0.075 & 0.035 \\
\hline
\end{tabular}

asymmetrical increase was found in the lateral parts of the DC's, presumably due to some rotation of the spinal cord.

The absolute values of the calculated fiber diameter distributions of the five models are rather different, particularly model A2 has small values. These differences could be caused by incorrect estimations of the distance between spinal cord and epidural electrode from the CT scans. Although the electrode positions are shown clearly, the circumference of the spinal cord is rather vague. Moreover, the CT scans have a limited resolution $(\approx 0.5 \mathrm{~mm})$. Therefore, the influence of an incorrect estimation of the electrode to spinal cord distance was estimated with the volume conductor model by changing the spinal cord position in the spinal canal by $0.3 \mathrm{~mm}$ laterally and $0.3 \mathrm{~mm}$ dorsally. The lateral change resulted in an average fiber diameter change of only $3 \%$, whereas moving the spinal cord $0.3 \mathrm{~mm}$ dorsally resulted in an average decrease of fiber diameter of $22 \%$.

The dorsoventral position of a fiber in the DC's significantly influences its stimulation threshold, as shown in Fig. 4. Here, a $10-\mu \mathrm{m}$ fiber was positioned at an increasing depth medially in the DC's, and the threshold stimulus was determined for TTL stimulation. In two models, having different thicknesses of the dorsal CSF layer (1.0 and $3.5 \mathrm{~mm}$ ), the calculated threshold doubled when the fiber was moved from the DC border to a depth of $0.4-0.5 \mathrm{~mm}$. As expected, the largest increase of threshold was found in the model with the smallest dorsal CSF layer [2]. To test the influence of distribution of fiber positions used in this study (Fig. 2), the calculations of patient model B1 were repeated with equidistant fiber positions, spanning the same width in each dorsal column. It was computed that medially the fiber diameters were increased up to $4 \%$, whereas laterally an increase of $6 \%-10 \%$ was found, except for the most lateral (abdominal) fiber, which still had the same position. Therefore, the spatial fiber diameter distribution, showing larger differences in the lateral than in the medial DC's, is not primarily caused by the distribution of their positions.

In order to evaluate differences of the calculated fiber diameters statistically, the data from all five models were combined. All fiber diameters calculated from each patient model were normalized to the average of the two diameters of the left and right posterior thigh. The resulting means and standard deviations of the entire study are shown in Fig. 5. The low-back and abdominal fibers were not incorporated because only few data were available, whereas in several cases these fibers were presumably DR fibers. The increase of the average fiber diameter from the medial to the lateral DC's is approximately $30 \%$. The increase at the left side differs somewhat from the right side. 


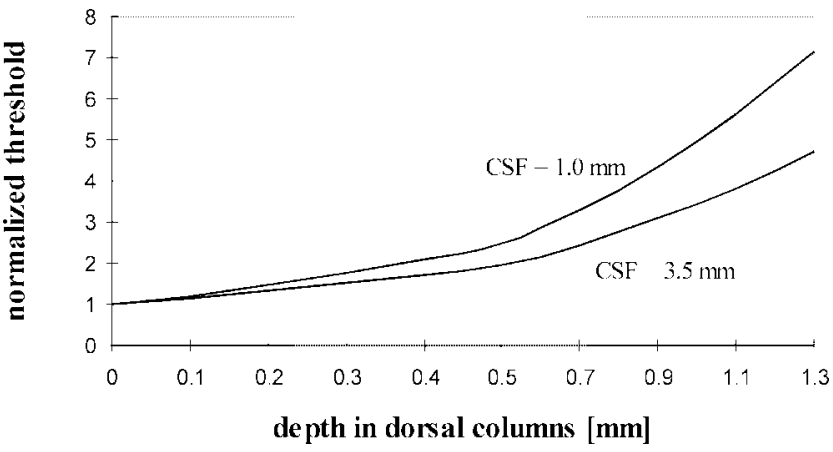

Fig. 4. Normalized stimulation threshold as a function of depth of a $10-\mu \mathrm{m}$ fiber medially in the DC's for two models, having a $1.0-\mathrm{mm}$ and $3.5-\mathrm{mm}$ dorsal CSF layer; transverse tripolar stimulation (balance $=1.0$ ).

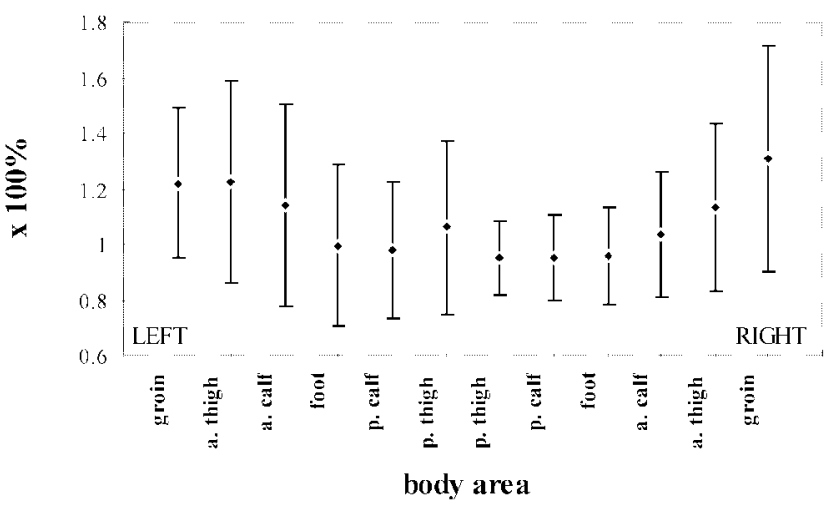

Fig. 5. Normalized fiber size distributions of all patient models; horizontally: body areas left and right; vertically: normalized mean fiber diameters and standard deviations.

The significance of the increase of the average fiber diameter from the medial to the lateral DC's was investigated using the Wilcoxon rank-sum test, because the number of data were too small to test for a normal distribution [31]. The normalized fiber diameters of the left and right groin are significantly larger than the more medial fiber diameters, except when compared with the (adjacent) anterior thigh fibers. The normalized diameters of the left- and right-posterior thigh do not differ significantly from fiber diameters as lateral as the anterior calf. From the tests, it can be concluded that there is a significant increase of fiber diameter from the medial to the lateral DC's. When comparing the normalized diameter distributions of corresponding fibers at the left and right side, it was found that for all six fibers the distributions left and right did not differ significantly. In addition, the overall trend from medial to lateral was investigated by averaging the right and left data, and comparing the best fitting linear and quadratic curves, as shown in Fig. 6. The second-order polynomial fit correlated better with the data (correlation coefficient: 0.98) than the linear fit (correlation coefficient: 0.90). Thus, it is not just shown that there is a significant increase of fiber diameter, but also that this increase is mainly restricted to the lateral parts of the DC's.

\section{DISCUSSION}

A statistically significant difference was found between the size of the largest dorsomedial and the largest dorsolateral

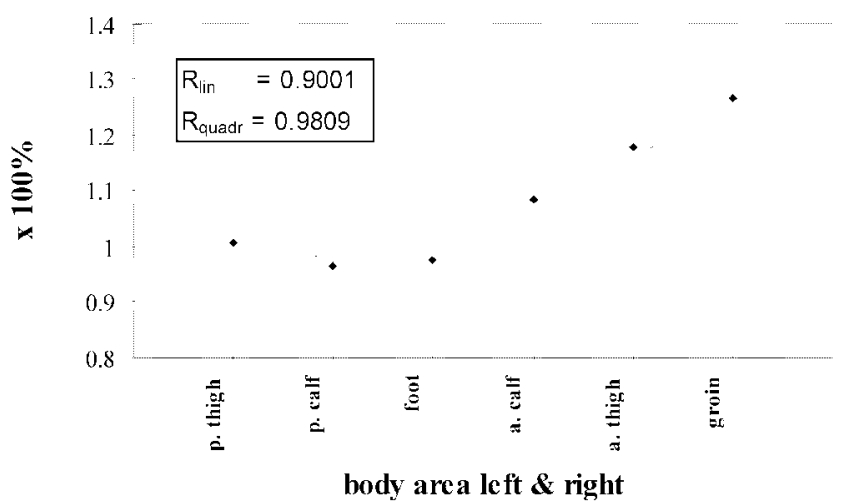

Fig. 6. Quadratic and linear fit of the mean normalized fiber size data of all patient models (average of left and right side); inset: correlation coefficients of both curves.

fibers in the superficial DC's at low-thoracic spinal cord levels (T7-T10). The mediolateral increase in fiber diameter of approximately $30 \%$, as shown in the current study, corresponds to the range reported from animal studies [16]-[20]. Preliminary results of a morphometric study of the human T10 segment indeed indicate that the mean diameter of DC fibers larger than $7 \mu \mathrm{m}$ decreases about $12 \%$ from lateral to medial (Feirabend, Choufoer, and Holsheimer, unpublished results). As expected, the calculated fiber diameter increase is mainly confined to the lateral DC's. After bifurcation of the DR fiber into a descending and an ascending DC fiber branch, collaterals are issued into the gray matter, which also causes a reduction of the fiber diameter [18]. This collateral branching occurs most frequently at the first few $\mathrm{cm}$ from the bifurcation point [21]. When it is considered that the DC fibers enter the DC's laterally and migrate in a medial direction when ascending to the brain, the diameter reduction (from lateral to medial) will be mainly confined to the lateral DC's. The range of calculated fiber diameters differed among patient models, whereby model A2 had very small diameters. The values calculated from the other models are well within the range of diameters determined in morphometric studies on human material [22]-[24].

The positions of fibers were assumed to be at the surface of the DC's. Although the general topography of the DC's is well known, the precise mediolateral positions of fibers corresponding to any body segment are not. Based on morphological data, we estimated fiber positions, corresponding to the considered body areas (see Fig. 2) at increasing separation from medial to lateral at the border of the DC's. Nevertheless, when an equidistant distribution was applied, a similar trend for the fiber diameters was found. Therefore, this trend in the mediolateral fiber size distribution does not originate from a specific mediolateral distribution of fiber positions.

Several aspects may have caused a bias in the results, and hereto explain the differences between the ranges of calculated diameters of the patient models. Although the average fiber diameter is smaller at higher vertebral levels [15], it is assumed that this does not strongly influence the results of this study, since only levels T7-T10 were considered.

The limited resolution of the CT scans may have led to a wrong estimation of the spinal cord position. Although the 
pre- and post-operative scans were combined to obtain an optimal picture of the spinal cord area, it was shown that dorsoventral changes of the position of the spinal cord as small as $0.3-\mathrm{mm}$ change threshold voltages by more than $20 \%$. This could have biased the calculated fiber diameters, as shown by the large difference of the diameter range in Fig. 3(a) and (b), both at the same spine level in the same patient. In contrast, little difference is seen between Fig. 3(c) and (d), also from one patient. A slightly incorrect mediolateral position of the spinal cord will not significantly influence the calculated fiber diameters, which agrees to previous results [2]. It was determined that the diameter values will only vary $3 \%$ when the fiber position is $0.3 \mathrm{~mm}$ more medially or laterally. In addition, there was presumably some difference in the position of the patient during CT scanning and testing, even though in both situations the patient was in a supine position. It can be concluded, that the dorsoventral position of the spinal cord in the dural sac mainly determines the range of calculated fiber diameters and that accurate distances between the electrode and the border of the DC's could not be determined. Therefore, the fiber diameters are only rough estimates, but the mediolateral diameter ratios are more reliable.

The standard deviations in Fig. 3(a)-(e) and 5 show that a rather large stochastic intra- and interpatient variation in the calculated fiber diameters exists. This can be caused by estimation of the threshold values of paresthesia, since patients indicate the perception of paresthesia in body areas while the stimulus voltage is continuously increased at a slow rate. Especially at low levels $(<0.5 \mathrm{~V})$, threshold estimates can be too high and will cause fiber diameter estimates being too small, although these low thresholds were only present in some body areas of patient A1. Moreover, small movements of the patients during the test sessions, and the fact that diameters were obtained from several contact configurations (using a single-turn potentiometer for the balance settings) for every patient, may also both contribute to the observed intrapatient variation in fiber diameters.

DR fiber stimulation is assumed when the calculated diameter of the fibers in the most lateral DC's strongly exceeds the diameters of the neighboring DC fibers, as shown in Fig. 3(a)-(c). The calculated DR fiber diameters will be smaller, because DR fibers generally need lower stimulus voltages for their excitation than DC fibers at the same (lateral) position [3]. In the models corresponding to Fig. 3(b)-(c), the DR fiber diameters were approximately $34 \%$ smaller than the corresponding DC fiber diameters and generally fitted well in the trends shown in these figures. The treatment of low-back pain is the main application of SCS in pain management [32]. However, low-back paresthesia was only seen in a few cases. In model B1 (left side) it is presumably related to DR stimulation, whereas in models A1 and C1 (left side) the calculated diameter is approximately 50\% smaller than expected at that position. This might indicate that the corresponding fibers are deeper in the DC's, having diameters as expected (cf. Fig. 4).

Finally, implementation of the results from this study can improve the UT-SCS model and future modeling studies can benefit from it. A realistic fiber distribution, both as to functional association and as to fiber diameters, will improve the capability to predict the recruitment order of paresthesia areas. However, more data from similar patient modeling are needed to obtain reliable estimates of fiber diameters. The calculated values should be validated by human morphometric data, which are, however, not yet available. But still it should be considered that, in order to predict the recruitment order of paresthesia, correct ratios are more important than correct absolute fiber diameter values.

\section{REFERENCES}

[1] R. Melzack, P. D. Wall, "Pain mechanisms: A new theory," Sci., vol. 150, pp. 971-979, 1965.

[2] J. J. Struijk, J. Holsheimer, G. G. van der Heide, H. B. K. Boom, "Recruitment of dorsal column fibers in spinal cord stimulation: Influence of collateral branching," IEEE Trans. Biomed. Eng., vol. 39, pp. 903-912, 1992.

[3] J. J. Struijk, J. Holsheimer, H. B. K. Boom, "Excitation of dorsal root fibers in spinal cord stimulation: A theoretical study," IEEE Trans. Biomed. Eng., vol. 40, pp. 632-639, 1993.

[4] J. J. Struijk, J. Holsheimer, "Transverse tripolar spinal cord stimulation: theoretical performance of a dual channel system," Med. Biol. Eng. Comput, vol. 34, pp. 273-279, 1996.

[5] J. Holsheimer, J. J. Struijk, N. R. Tas, "Effects of electrode geometry and combination on nerve fiber selectivity in spinal cord stimulation," Med. Biol. Eng. Comput., vol. 33, pp. 676-682, 1995.

[6] J. Holsheimer, W. A. Wesselink, "Optimum electrode geometry for spinal cord stimulation: the narrow bipole and tripole," Med. Biol. Eng. Comput., vol. 35, pp. 493-497, 1997.

[7] R. A. Davidoff, "The dorsal columns," Neurol., vol. 39, pp. 1377-1385, 1989.

[8] G. Werner, B. L. Whitsel, "The topology of dermatomal projection in the medial lemniscal system," J. Physiol., vol. 192, pp. 123-144, 1967.

[9] A. E. Walker, T. A. Weaver, "The topical organization and termination of the fibers of the posterior columns in macaca mulatta," J. Comp. Neurol., vol. 76, pp. 145-158, 1942.

[10] Y. Hwang, E. J. Hinsman, O. F. Roesel, "Caliber spectra of fibers in the fasciculus gracilis of the cat cervical spinal cord: A quantitative electron microscopic study," J. Comp. Neurol., vol. 162, pp. 195-204, 1975.

[11] T. Hongo, N. Kudo, S. Sasaki, M. Yamashita, K. Yoshida, N. Ishizuka, H. Mannen, "Tracjectory of group Ia and Ib fibers from the hind-limb muscles at the L3 and L4 segments of the spinal cord of the cat," $J$. Comp. Neurol., vol. 262, pp. 159-194, 1987.

[12] B. L. Whitsel, L. M. Petrucelli, G. Sapiro, H. Ha, "Fiber sorting in the fasciculus gracilis of squirrel monkeys," Exp. Neurol., vol. 29, pp. 227-242, 1970 .

[13] J. L. Culberson, P. B. Brown, "Projections of hindlimb dorsal roots to lumboscaral spinal cord of cat," J. Neurophysiol., vol. 51, pp. 516-528, 1984.

[14] M. C. Smith, P. Deacon, "Topographical anatomy of the posterior columns of the spinal cord in man: The long ascending fibres," Brain, vol. 107, pp. 671-698, 1984.

[15] J. L Culberson, B. C. Albright, "Morpohologic evidence for fiber sorting in the fasciculus cuneatus," Exp. Neurol., vol. 85, pp. 358-370, 1984.

[16] D. Petit, P. R. Burgess, "Dorsal column projection of receptors in cat hairy skin supplied by myelinated fibers," J. Neurophysiol. Sci., vol. 31, pp. 849-855, 1968

[17] A. G. Brown, "Cutaneous afferent fibre collaterals in the dorsal column of the cat," Exp. Brain Res., vol. 5, pp. 293-305, 1968.

[18] K. W. Horch, P. R. Burgess, D. Whitehorn, "Ascending collaterals of cutaneous neurons in the fasciculus gracilis of the cat," Brain Res. vol. 117, pp. 1-17, 1976.

[19] R. Fern, P. J. Harrison, J. S. Riddell, "The dorsal column projection of muscle afferent fibers from the cat hindlimb," J. Physiol., vol. 401, pp. 97-113, 1988.

[20] P. D. Wall, P. Shortland, "Long-range afferents in the rat spinal cord I: Numbers, distances and conduction velocities," Phil. Trans. R. Soc. Lond., vol. 334, pp. 85-93, 1991.

[21] R. E. W. Fyffe, Afferent fibers, in Handbook of the Spinal Cord, vols 2 and 3, Anatomy and Physiology, R. A. Davidoff, Ed. New York, Marcel-Dekker, pp. 79-136, 1984.

[22] G. Häggqvist, "Analyse der Faserverteilung in einem Rückenmarkquerschnitt (Th 3)," Zeitschrift f. Mikr.-Anat. Forschung, vol. 39, pp. 1-34, 1936. 
[23] A. Ohnishi, P. C. O’Brien, H. Okazaki, P. J. Dyck, "Morphometry of myelinated fibers of fasciculus gracilis of man," J. Neurol. Sci., vol. 27, pp. 163-172, 1976.

[24] M. Makino, K. Mimatsu, H. Saito, N. Konishi, Y Hashizume, "Morphometric study of myelinated fibers in the human cervical spinal cord white matter," Spine, vol. 21, pp. 1010-1016, 1996.

[25] J. Holsheimer, B. Nuttin, G. W. King, W. A. Wesselink, J. M. Gybels, P. de Sutter, "Clinical evaluation of paresthesia steering with a new system for spinal cord stimulation," Neurosurg., vol. 42, pp. 541-547, 1998.

[26] G. Dahlquist, O. Björk, Numerical Methods. Englewood Cliffs, NJ: Prentice-Hall, 1974.

[27] J. J. Struijk, J. Holsheimer, G. Barolat, J. He, H. B. K. Boom, "Paresthesia thresholds in spinal cord stimulation: A comparison of theoretical results with clinical data," IEEE Trans. Rehab. Eng., vol. 1, pp. 101-108, 1993.

[28] D. R. McNeal, "Analysis of a model for excitation of myelinated nerve," IEEE Trans. Biomed. Eng., vol. BME-23, pp. 329-337, 1976.

[29] S. Y. Chiu, J.M Ritchie, R. B. Rogart, D. Stagg, "A quantitative description of membrane currents in rabbit myelinated nerve," J. Physiol., vol. 292, pp. 149-166, 1979.

[30] J. Holsheimer, "Computer modelling of spinal cord stimulation and its contribution to therapeutic efficacy (Review)," Spinal Cord, vol. 36, pp. 531-540, 1998

[31] M. Hollander, D. A. Wolfe, Nonparametric Statistical Methods. New York: Wiley, 1973

[32] K. J. Burchiel, V. C. Anderson, F. D. Brown, R. G. Fessler, W. A. Friedman, S. Pelofsky, R. L. Weiner, J. Oakley, D. Shatin, "Prospective, multicenter study of spinal cord stimulation for relief of chronic back and extremity pain," Spine, vol. 21, pp. 2786-2794, 1996

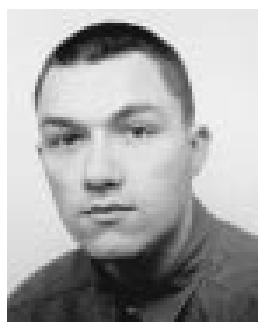

Wilbert A. Wesselink was born in Zelhem, the Netherlands, in 1970. In 1994, he received the M.Sc degree in electrical and biomedical engineering from the University of Twente, the Netherlands. He joined the Institute for Biomedical Technology in 1994 as a Ph.D. degree student.

His research interests are in computer modeling of spinal cord stimulation and the evaluation of clinical studies in which new electrode configurations are applied.

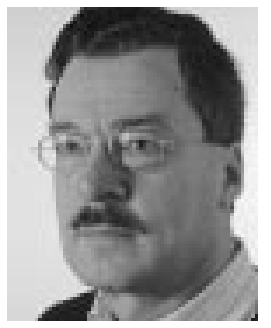

Jan Holsheimer was born in Enschede, the Netherlands, in 1941. He received the M.Sc. degree in biology and biophysics from the University of Groningen, the Netherlands, in 1965, and the Ph.D. degree in biomedical engineering from the University of Twente, the Netherlands, in 1982.

In 1965, he joined the Biomedical Engineering Department of Electrical Engineering at the University of Twente. His primary research interests are the theoretical, experimental, and clinical aspects of electrical stimulation of the nervous system. His studies are focused on the design of electrodes enabling the stimulation of specific neuronal pathways, as characterized by their positions and fiber diameters.

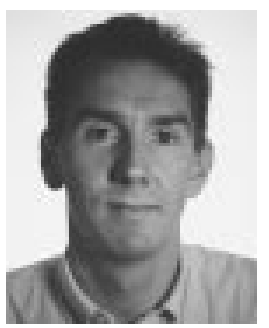

Bart Nuttin was born in Leuven, Belgium, in 1959. $\mathrm{He}$ received the Ph.D. degree in medical sciences in 1996.

He became a Neurosurgeon in 1991. Since 1996, he has been responsible for stereotactic and functional neurosurgery at the University Hospitals in Leuven, Belgium. He performs research in the Lab of Experimental Neurosurgery and Neuroanatomy and teaches in medical school at the Catholic University of Leuven (K.U. Leuven), Belgium. His research is on neuromodulation and on injury to the central nervous system.

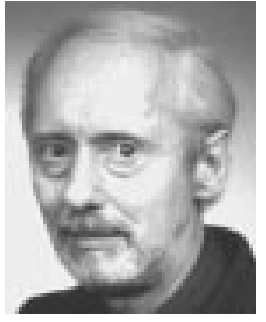

Herman B. K. Boom (A'89-SM'97) was trained as a Medical Physicist at the University of Utrecht, The Netherlands, where he received the $\mathrm{PhD}$ degree in 1971.

He joined the Department of Medical Physics and Medical Physiology where he was engaged in research in the field of cardiac mechanics and taught physiology and biophysics. Since 1976, he has been a Professor of Medical Electronics in the Department of Electrical Engineering, University of Twente, The Netherlands. His research interests are in cardiovascular system dynamics, bioelectricity, and rehabilitation technology.

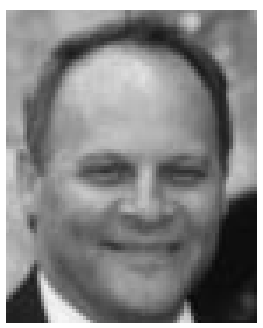

Gary W. King received the B.Sc. degree in physics in 1969 , the M.Sc. degree in nuclear physics in 1973, and the Ph.D. degree in neurophysiology in 1978, all at the University of Minnesota, Minneapolis.

After postdoc research in autonomic and pain neural systems, he worked on urological prostheses for nine years. He is a Clinical Research Scientis at Medtronic Neurological, Minneapolis, MN. Since 1989, he has designed and managed clinical studies of spinal cord stimulation for chronic pain and peripheral vascular disease and of deep brain stimulation for pain and tremor.

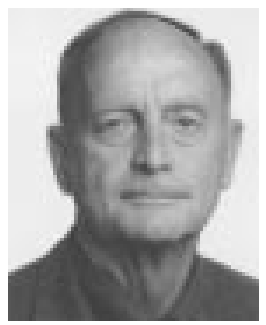

Jan M. Gybels is a Belgium native. After obtaining the medical degree at the University of Leuven (K.U.L.), Leuven, Belgium, in 1953, he was trained in general surgery in Leuven, in neurosurgery at the Montreal Neurological Institute, Montreal, Canada, and in neurology at the National Hospital, Queen Square, London. In 1962, he obtained the Ph.D. degree at the University of Leuven.

He has served as Professor of neurology and neurosurgery, Chief of clinic of neurosurgery and Director of the Laboratory of experimental neurology at the University of Leuven. In 1994, he became Professor Emeritus. His main interests have been focused on functional neurosurgery, and more particularly pain, abnormal movements, and psychiatric disorders. The most important new techniques in his research have been microneurography, supraspinal neurostimulation, and the integration of modern computer techniques in stereotactic neurosurgery. His long experience of pain surgery has been synthesized in Neurosurgical Treatment of Persistent Pain, with W. Sweet (Basel, Switzerland: Karger, 1989).

Dr. Gybels is board certified in general surgery, neurosurgery, and neurology. He is a member of the Royal Academy of Medicine of Belgium.

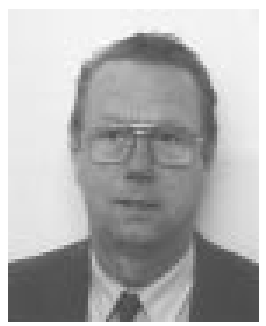

Paul de Sutter was born in Scheldewindeke, Belgium, in 1943. He received the degree of technical Engineer A1 from the R.H.T.H.S. of Gent, Begium.

In 1968, he worked in the technical Engineering Department of the University Hospital AZ St. Rafal, Leuven, Belgium. Since 1969, he has worked in the Research Department of Neurology and Neurosurgery at the University of Leuven and the University Hospital UZ Gasthuisberg Leuven, Belgium. 